\title{
Apuntes fenomenológicos sobre el problema del yo y la conciencia en la filosofía de Sartre
}

Arturo Alberto Cardozo Beltrán

Universidad del Atlántico, Colombia 


\title{
Apuntes fenomenológicos sobre el problema del yo y la conciencia en la filosofía de Sartre*
}

Resumen: en este artículo se hace una revisión del problema que surge en la primera parte de La Trascendencia del Ego escrita por el filósofo francés Jean Paul Sartre. Tal problemática versa en que si es posible que la categoría "yo" habite en el interior de la conciencia. Esta indagación revelara la tesis sartriana sobre la conciencia, la cual entiende a la conciencia como exterioridad pura, es decir en su interior no habita ninguna instancia metafísica, como lo podría ser el yo, el cogito, el inconsciente etc. Continuamente se argumentará la imposibilidad e inconsistencia de que estas estructuras metafísicas habiten en el interior de la conciencia.

Palabras claves: conciencia, yo, moi, cogito, Sartre.

\section{Phenomenological notes about the problem of self and consciousness in the Sartre's philosophy}

\begin{abstract}
: this article is a review of the problem that emerges from the first part of Transcendence of the Ego written by Jean-Paul Sartre. The basis of this problem is about the ego possibility of life inside of the conscience. This thinking will reveal the Sartrean thesis about consciousness, which understands consciousness as pure exteriority, that's means, no metaphysical entity inhabits its interior, as it could be the self, the cogito, the unconscious etc. The impossibility and inconsistency of these metaphysical structures inhabiting the interior of consciousness will be continually argued.
\end{abstract}

Keywords: conscience, the self, moi, cogito, Sartre.

Fecha de recepción: 26 de junio de 2018

Fecha de aceptación: 19 de noviembre de 2018

Forma de citar (APA): Cardozo-Beltrán, A. (2019). Apuntes fenomenológicos sobre el problema del yo y la conciencia en la filosofía de Sartre. Revista Filosofía UIS, 18(1), doi: http://dx.doi.org/10.18273/revfil.v18n1-2019005

Forma de citar (Harvard): Cardozo-Beltrán, A. (2019). Apuntes fenomenológicos sobre el problema del yo y la conciencia en la filosofía de Sartre. Revista Filosofía UIS, 18(1),109128.

Arturo Alberto Cardozo Beltrán: colombiano. Magíster en Filosofía, profesor Universidad del Atlántico, Colombia.

Correo electrónico: arturocar2005@hotmail.com

ORCID: https://orcid.org/0000-0002-2905-6433

* Artículo de reflexión derivado de investigación. 


\section{Apuntes fenomenológicos sobre el problema del yo y la conciencia en la filosofía de Sartre}

En 1933 Sartre conoce la fenomenología husserliana por la lectura que hizo del libro de Emanuel Lévinas La teoría de la intuición en la fenomenología de Husserl, así como del libro de Georges Gurvitch Las tendencias actuales de la filosofía alemana, estas lecturas lo motivaron a irse a estudiar al Instituto francés de Berlín, allí Sartre comienza sus lecturas fenomenológicas: las obras publicadas de Edmund Husserl y la primera parte de Ser y Tiempo de Martin Heidegger. Su primera obra publicada como resultado de sus estudios fue la Trascendencia del Ego (Cfr. Suárez, 2016, p. 345). En este texto se esboza la tesis central de Sartre sobre la conciencia y su relación con el yo. Esta tesis entiende la conciencia como exterioridad pura, en el sentido en que ella no tiene contenido, en que no hay en ella seres metafísicos que la habiten como la idea del yo, del alma o del inconsciente. De este modo, Sartre toma distancia de autores que han creído que estructuras como el yo, el cogito, el moi, entre otras ${ }^{1}$, hacían "parte" de la conciencia, es decir, instancias que la integran o la conforman.

Para este trabajo se mostrará y analizará la argumentación que ofrece Sartre para justificar la exterioridad de la conciencia, es decir, que la conciencia es una "nada pura" o "contenido vacío", en la medida en que, su ser se encuentra siempre fuera de sí misma, es plena trascendencia. Así se entiende la afirmación de que solamente la conciencia es cuando es conciencia de algo ${ }^{2}$. Demostrar esto, en últimas, es para Sartre el primer paso de la filosofía:

\footnotetext{
${ }^{1}$ Los estados como el amor, el odio, el inconsciente.

2 Sartre aclara lo propio de la conciencia a partir de la concepción husserliana, que la concibe fundamentalmente como conciencia de algo, es decir como intencional. En un pasaje de El Ser y la Nada afirma Sartre: Toute conscience est conscience de quelque chose. Cette définition de la conscience peut être prise en deux sens bien distincts: ou bien nous entendons par là que la conscience est constitutive de l'être de son objet, ou bien cela signifie que la conscience en sa nature la plus profonde est rapport à un être transcendant. Mais la première acception de la formule se détruit d'elle-même: être conscience de quelque chose c'est être en face d'une présence concrète et pleine qui n'est pas la conscience. Sans doute peut-on avoir conscience d'une absence. Mais cette absence paraît nécessairement sur fond de présence. [...] La conscience est conscience de quelque chose: cela signifie que la transcendance est structure constitutive de la conscience; c'est-à-dire que la conscience naît portée sur un être qui n'est pas elle (1943, pp. 26-28).
} 
La première démarche d'une philosophie doit donc être pour expulser les choses de la conscience et pour rétablir le vrai rapport de celle-ci avec le monde, à savoir que la conscience est conscience positionnelle du monde. Toute conscience est positionne lle en ce qu'elle se transcende pour atteindre un objet, et elle s'épuise dans cette position même: tout ce qu'il y a d'inlenlion dans ma conscience actuelle est dirigé vers le dehors, vers la table; toutes mes activités judicatives ou pratiques, toute mon affectivité du moment se transcendent, visent la table et syy absorbent $^{3}$ (1943, p. 18).

Por tanto, la forma en la que se expondrá la tesis sartriana sobre la conciencia, para este trabajo se dividirá en tres partes: la primera parte corresponderá al desarrollo del problema del yo como un habitante de la conciencia, es decir se entablará la discusión que presenta Sartre con las interpretaciones posteriores al kantismo. Estas han manifestado, desde la interpretación de la filosofía kantiana, la inseparabilidad del yo con la conciencia. La segunda parte será la exposición del cogito como conciencia reflexiva, esta es la propuesta sartriana que pretende hacer la comunicación entre el cogito y la res extensa, mediante la exposición de la conciencia reflexiva y la conciencia irreflexiva. Finalmente, la tercera parte será la discusión que hace Sartre con la teoría del "mí mismo", esta teoría es la propuesta, donde se afirma que, todo acto realizado por el individuo se debe a un impulso egoísta originado en el interior de la conciencia. Todo lo anterior se hará con el objetivo de sustentar la veracidad de la tesis sartriana y su aporte a la filosofía contemporánea.

\section{Teoría de la presencia formal del Yo}

Sartre (1990) dice en La Trascendencia del Ego que "el Ego no está ni formal ni materialmente en la conciencia: está fuera, en el mundo; es un ser del mundo, como también lo es el Ego del prójimo" (p. 29). ¿Qué implica que el ego sea un ser en el mundo? ¿Cómo argumenta Sartre dicha afirmación? Inicialmente comienza citando un fragmento de la segunda parte de la Crítica de la Razón Pura, titulada "Analítica trascendental", en donde Kant (2011) dice: "El yo pienso tiene que poder acompañar a todas mis representaciones" (p. 175). A partir de esta afirmación Sartre (1990) se preguntará: “iHay que concluir de ello que, de hecho, habite un Yo todos nuestros estados de conciencia y efectúe realmente la síntesis suprema de nuestra experiencia?" (p. 32). En otras palabras: ¿es el yo un polo unificador de todos nuestros estados de conciencia? ¿El yo siempre está detrás de cada estado de conciencia? En la Crítica de la Razón Pura Kant (2011) dirá que si

\footnotetext{
${ }^{3}$ El primer paso de una filosofía ha de ser, pues expulsar las cosas de la conciencia y restablecer la verdadera relación entre ésta y el mundo, a saber, la conciencia como conciencia posicional del mundo. Toda conciencia es posicional en cuanto se trasciende para alcanzar un objeto, y se agota en esa posición misma: todo cuanto hay de intención en mi conciencia actual está dirigido hacia el exterior, hacia la mesa; todas mis actividades judicativas o prácticas, toda mi afectividad del momento, se trascienden, apuntan a la mesa y en ella se absorben (Sartre, 1984, p. 21).
} 
el yo no acompaña a todas nuestras representaciones "no sería representado en mí algo que no podría ser pensado" (p. 175). Esto quiere decir que si el yo pienso no acompaña mi representación, ella no podría ser pensada o sería imposible su representación. Como se sabe, Kant busca con el descubrimiento del yo trascendental ganar la instancia que garantice el conocimiento del mundo, de lo que se puede conocer. ¿Sucederá lo mismo para la conciencia? ¿Es imposible que existan momentos de la conciencia sin el yo?

Para Sartre no es necesario que el yo sea un compañero inseparable de cada estado de conciencia; esto se debe a que para el filósofo francés el problema de la crítica ${ }^{4}$ es un problema de derecho ${ }^{5}$ y no de hecho. Kant no afirma nada sobre la existencia del hecho del yo pienso, a modo de que se puedan determinar las condiciones de posibilidad de la experiencia del yo (Sartre, 1966, p. 14). Esto se constata en Crítica de la Razón Pura cuando Kant (2011) dice: "Todo múltiple de la intuición ${ }^{6}$ tiene una relación necesaria con el yo pienso, en el mismo sujeto donde ese múltiple es hallado" (p. 176). Esto quiere decir que toda representación antes de ser pensada surge del sujeto, y por tanto puedo considerar como mías mis representaciones. En este apartado de la Crítica de la Razón Pura, Kant está tratando el problema del yo pienso desde una cuestión de derecho y no desde una cuestión de hecho, pues está estudiando las condiciones de posibilidad para que se dé la experiencia de representación del yo desde lo a priori o, en otras palabras, desde la deducción trascendental. En últimas, es lo dicho por Sartre; Kant está determinando las condiciones de posibilidad de la experiencia desde una cuestión de derecho, pues al determinar cuáles son las condiciones en las que yo pueda considerar como míos mi percepción o mi pensamiento, pertenece a una cuestión de derecho (Cfr. Sartre, 1966, p. 14). Además, para Kant la representación que puede darse antes de todo pensar (intuición) se da por un acto de espontaneidad y no pertenece a la sensibilidad, o en palabras de Sartre, no pertenece a una

\footnotetext{
${ }^{4}$ El problema de la Crítica de la Razón Pura. Es decir, Kant somete a crítica la razón, en los términos de fijar los límites de lo que puede ser conocido, dejando a un lado la pregunta por la "cosa en sí" para centrar el análisis en la forma en que el ser humano conoce. El problema de la crítica es por ello en Kant un problema especialmente epistemológico.

${ }^{5}$ Immanuel Kant en la segunda parte, "Analítica trascendental", de la Crítica de la Razón Pura va a utilizar los términos quid juris y quid facti (propios de la ciencia del derecho), que en latín traducen 'cuestión de derecho' y 'cuestión de hecho', para explicar la deducción trascendental y la deducción empírica (Cfr. Kant, 2011, pp.165-167). Entre todos los conceptos que constituyen la trama abigarrada del conocimiento humano existen algunos que son independientes de toda experiencia -a priori- para su constatación o legitimación con respecto a la experiencia, es necesario que pasen por la deducción trascendental, ya que esta es la explicación del modo como esos conceptos a priori puedan referirse a lo real. Además, se distingue de la deducción empírica, ya que en esta se refiere al modo como un concepto ha sido adquirido mediante la experiencia y la reflexión, es decir, la deducción empírica no refiere a la legitimidad del concepto sino a su factum en otras palabras se refiere directamente a la constatación desde el hecho (Cfr. 2011, p. 166).

${ }^{6}$ Para Kant (2011) la intuición es "la representación que pueda ser dada antes de todo pensar" (p. 176).
} 
cuestión de hecho. Kant denomina este tipo de representación apercepción pura ${ }^{7}$, y declara que es para distinguirla de la empírica; la razón de ello es:

Porque es aquella autoconciencia que, produciendo la representación yo pienso (que tiene que poder acompañar a todas las demás y que es una y la misma en toda conciencia), no puede ser deducida de ninguna otra. A su unidad doy el nombre de unidad trascendental de la autoconciencia para señalar la posibilidad del conocimiento a priori nacido de ella (Kant, 2011, p. 176).

Con esta cita se observa que Kant, según Sartre, no se preocupó por el modo como se constituye de hecho la conciencia empírica, ni mucho menos la dedujo de una conciencia superior o de una hiperconciencia constituyente. En definitiva, la conciencia trascendental es para Kant el conjunto de las condiciones necesarias para que exista una conciencia empírica (Cfr. Sartre, 1966, p. 15).

Se debe afirmar que no obstante la crítica de Sartre a Kant, el haber ignorado en su análisis del yo la situación de hecho, concede el filósofo francés que el yo Pienso debe poder acompañar todas nuestras representaciones desde una cuestión de derecho; ipero las acompaña de hecho? El yo que encontramos en nuestra conciencia, ise hace posible por la unidad sintética de nuestras representaciones, o bien es él el que unifica de hecho, unas con otras, las representaciones? ¿Qué significa para la conciencia que el yo acompañé o no nuestras representaciones? ¿Determina el yo el ser de la conciencia? ¿Qué es, en últimas, el yo para Sartre? (Cfr. Sartre, 1966, pp. 15-16).

Para resolver todos estos interrogantes Sartre encuentra en la fenomenología de Husserl un buen camino, pues dirá que para resolver el problema de hecho del yo en la conciencia será necesario recurrir a Husserl, ya que para Sartre la fenomenología propuesta por Husserl es una ciencia de hechos, y sus problemas planteados son de hechos ${ }^{8}$. Por lo tanto, para que la conciencia trascendental

\footnotetext{
"El origen histórico del término "apercepción", de donde Kant sin duda lo tomó, se haya en la filosofía de Leibniz. A la percepción, por ejemplo, nuestro ver un paisaje, opone Leibniz la apercepción, en este caso, el darnos cuenta que estamos viendo un paisaje. También en otro sentido "apercepción" equivale, para Leibniz, a "conciencia de sí mismo" (Nota del traductor, 2011, p. 176).

${ }^{8}$ Cabe aclarar que Husserl (2013) en Ideas I pareciera contradecir lo que acaba de ser expuesto, pues cuando explica la diferencia entre psicología y fenomenología pura o trascendental dice: "Aqu se fundara la fenomenología pura o trascendental no como una ciencia de hechos, sino como una ciencia de esencias (como una ciencia eidética; como una ciencia que quiere llegar exclusivamente a "conocimientos esenciales" y no fijar, en absoluto, hechos" (p. 9). Sin embargo, Sartre (1990) explica en una nota a pie de página en La Trascendencia del Ego que la ciencia de hechos viene a ser lo mismo que ciencia de esencias, en el contexto que se está trabajando (Cfr. p. 35), puesto que las esencias son esencias de hechos y no de seres puros. Se debe añadir que en el existencialismo sartriano se denota una clara diferenciación entre la existencia y la esencia, donde la primera es la primacía en el estudio existencialista (Cfr. Koffi, 2013, p. 81).
} 
de Kant se convierta en un problema de hecho y no solo de derecho, el filósofo francés dirá que la solución se encuentra en la epojép propuesta por Husserl: "La conscience transcendantale de Kant, Husserl la retrouve et la saisit par غ̇ं̄o $\eta^{10 "}$ (Sartre, 1966, p. 35).

Además, Sartre está de acuerdo con Husserl respecto a la conciencia trascendental como constituyente del mundo. Encuentra en la concepción husserliana de la conciencia como conciencia trascendental la idea de un ego como parte constitutiva del propio mundo. En Husserl la conciencia es trascendental por ser siempre ella misma conciencia de algo, es decir, por ser conciencia intencional, ya en contacto con el mundo, y no porque su ser trascendente este definido por los actos de un yo que quiere conocer el mundo, como si este, el mundo, existiera fuera de él. Si la conciencia es para Husserl conciencia "constituyente" del sentido del mundo, lo es ante todo porque ella es conciencia del mundo, porque este es inherente a ella, en la medida en que no habría conciencia sin la existencia fáctica del mundo. La conciencia "constituyente" no crea, en sentido estricto, el sentido del mundo, ya que ella es un ser del mundo, un ser trascendente. Pero

\footnotetext{
${ }^{9}$ Una filosofía sin supuestos, es decir, un conocimiento filosófico universalmente válido en todas partes y en todos los tiempos, es el imperativo categórico de Husserl, y hasta es el lema de su escuela (Cfr. Vélez, p. 7). Para alcanzar dicho propósito, Husserl propone la reducción fenomenológica. Y esta consiste en un primer momento en realizar la epojé, término que significa "abstención o suspensión de juicio". Así describe Husserl (2013) el sentido de la epojé: "Ponemos fuera de acción la tesis general inherente a la esencia de la actitud natural; ponemos entre paréntesis todo lo que ella abarca ónticamente: así pues, este mundo natural entero, que está constantemente "para nosotros ahí", "ahí delante", y que seguirá estándolo incesantemente como "realidad" de que tenemos conciencia, aunque nos dé por ponerlo entre paréntesis" (p. 144). Es decir, la operación "crítica" que consiste en la purificación cabal de las opiniones, prejuicios, ideas heredadas o espontáneas que siempre hemos aceptado sin dudar sobre su veracidad. Es la puesta entre "paréntesis" de todas nuestras creencias mientras no puedan presentarse como una evidencia innegable (Cfr. Villoro, citado en Montero, 2007, p. 137). Cuando se da la abstención del juicio y el mundo queda entre paréntesis, pasamos de una actitud natural o ingenua (en esta no existe ninguna reflexión filosófica) a una actitud fenomenológica. Es decir, todo lo que pertenecía a la actitud natural, el mundo físico, la cultura, la religión; en palabras de Husserl (2013), ha "sucumbido a la desconexión" (p. 208). Sin embargo, aún la reducción no se ha dado por completo, pues el mismo Husserl en Ideas, en la segunda parte del capítulo cuatro, consagrado totalmente a la reducción, se pregunta qué elementos han quedado después de la epojé y cuáles se deben poner entre paréntesis. Se concluirá diciendo que la reducción fenomenológica no es un proceso sencillo, ya que trae ciertas dificultades, Husserl las señala cuando afirma: "Se presentan dificultades en un punto límite. El hombre como ser de la naturaleza y como persona en el conglomerado personal, en el de la "sociedad", es desconectado; igualmente todo ser animal. Pero ¿Qué pasa con el yo puro? ¿También el yo fenomenológico con el que nos encontramos se ha convertido mediante la reducción fenomenológica en una nada trascendental?" (2013). En definitiva "La reducción fenomenológica no es un proceso simple, puede verse desde diferentes aspectos que expresan pasos distintos. Es, a la vez, paso de lo simplemente mentado a lo dado en la evidencia, del ser relativo al ser absoluto, del mundo como suma de realidades a su origen constituyente y fuente de sentido, del yo perdido en el mundo al yo consciente de sí mismo. Con el mismo término Husserl designa un complejo cambio de actitud que puede mostrar una u otra faceta, sin agotarse en ninguna" (Villoro, citado en Montero, 2007, p. 137).
}

${ }^{10}$ Epojé. 
en tal análisis ¿cómo resuelve Husserl el problema del yo como un acompañante de todas nuestras representaciones? Sartre (1966) dice que Husserl responde al problema: "Après avoir considéré que le Moi était une production synthétique et transcendante de la conscience" (p. 20). Sin embargo, Sartre afirma que Husserl incurre en una posición idealista de la conciencia, al regresar, particularmente en su libro Ideas I, a la teoría clásica de un yo trascendental que estaría detrás de cada conciencia. Esto lo sustenta Sartre cuando afirma:

d'un Je transcendantal qui serait comme en arrière de chaque conscience, qui serait une structure nécessaire de ces consciences, dont les rayons (Ichstrahl) ${ }^{11}$ tomberaient sur chaque phénomène qui se présenterait dans le champ de l'attention ${ }^{12}$ (1990, p. 37).

La existencia de un yo trascendental "detrás" de cada conciencia, esto es, como el fundamento que irradiaría todo el campo de la conciencia con su luz, se justifica por la necesidad que tiene la conciencia de esta unidad e individualidad. Empero, Sartre dice que la fenomenología no necesita recurrir a este yo, dado que, al ser la conciencia "conciencia intencional", se trasciende a sí misma en cuanto que está se dirige hacia el mundo. La "unidad" de la conciencia no está, entonces, determinada por ella misma, desde un yo como lugar de su articulación, sino que es unidad con el mundo, de lo que ella es conciencia. La unidad de la conciencia, en otras palabras, está en que ella es conciencia de "algo", de un objeto real o abstracto. El objeto termina siendo la unificación de la conciencia. Lo termina siendo porque como dice Sartre (1966): "L'unité des mille consciences actives par lesquelles j'ai ajouté, j'ajoute et j'ajouterai deux à deux pour faire quatre, c'est l'objet transcendant "deux et deux font quatre »" (p. 21). En este punto cuando Sartre se refiere a la unidad de las mil conciencias, está hablando de todas las vivencias que tuvo mi conciencia en el pasado para llegar al objeto trascendente, que en este caso es "dos más dos son cuatro". Al apuntar mi conciencia a este objeto trascendente, hace que él sea el principio de unificación, pues como se ha dicho, la conciencia es intencional, apunta a un ser que no es ella misma, para terminar, unificándose con el objeto trascendente.

Para que la conciencia no necesite del yo como principio de unificación, aún queda un problema por resolver, y es el problema del tiempo. Dicho problema consiste en que se necesita un principio de unidad, con respecto a las conciencias del pasado con las del presente, para que exista un continuo flujo que sea susceptible de poner fuera de él objetos trascendentales. Sartre resuelve este problema aludiendo que Husserl nunca recurrió al poder sintético del yo para resolver el problema del tiempo, ya que la conciencia se unifica así misma por

\footnotetext{
${ }^{11}$ Palabra escrita así en el original como un compuesto de las palabras alemanas Ich (yo) y Strahl (rayo). 12 "Un Yo trascendental que estaría como por detrás de cada conciencia; que sería una estructura necesaria de estas conciencias; y cuyo rayo (Ichstrahl) caería sobre cada fenómeno que se presenta en el campo de la atención" (Sartre, 1990, p. 37).
} 
medio de lo que llama Sartre (1966) un juego de intencionalidades "transversales" (p. 22). Esto quiere indicar que la conciencia en el momento de remitirse a sí misma no lo hace de manera consecutiva, es decir, que no aparece una conciencia primero y luego la otra; por el contrario, la forma en la que la conciencia se remite a sí misma es que varías conciencias aparecen al mismo tiempo remitiéndose a una sola conciencia; o en otras palabras, tanto las conciencias de experiencias pasadas como las conciencias en el presente se unifican en una sola conciencia.

Sartre (1966) concluye que el yo trascendental no tiene razón de ser, y peor aún: si existe un yo trascendental es equivalente a la muerte de la conciencia ( $p$. 23), puesto que para que la conciencia tome conciencia de sí es necesario que ella sea conciencia de un objeto trascendente y no conciencia como acto de un yo trascendental que se piensa solo a sí mismo. El "objeto" de la conciencia no está, entonces, constituido para ella misma a modo de un acto posicional, sino que él está, por su naturaleza de "objeto", fuera de ella. La conciencia es conciencia de sí en el momento que es conciencia de un objeto trascendente; ella es simplemente conciencia de ser conciencia de este objeto, y en esto radica el principio de la fenomenología: toda conciencia es conciencia de algo. Para Sartre lo que explica la unidad de la conciencia no es, de acuerdo con lo dicho, la existencia de un pensamiento reflexivo (cogito), propio de un yo trascendental, el cual realizaría la síntesis con el objeto pensado. Pero no niega, con esto, la existencia de un cogito, de un pensamiento, sino el hecho de que este se conciba como un pensar puro, enfrentado a sus propias cogitaciones, es decir, a sus propios pensamientos. El cogito sartriano trasciende su propio pensamiento (Cfr. Sartre, 1996, pp. 23-24).

Se concluye que al introducir el yo en la conciencia como interioridad se destruye la definición de esta anteriormente mencionada. Además se pierde el sentido tan profundo y original que Sartre está buscando para la conciencia, que la conciencia sea un "absoluto no sustancial"13. El yo para Sartre no es conciencia, ni una cualidad traslucida, ni un habitante de la conciencia. Para que la conciencia tenga este carácter que le pretende dar Sartre de absoluto, simplemente necesita ser conciencia de sí misma; empero, si el yo se convierte en una estructura

\footnotetext{
${ }^{13}$ Cabe aclarar que por "absoluto no sustancial" se entiende como lo explica Álvarez: lo propio de la conciencia es su ausencia de limitación o fundamentación, ya que ella misma constituye sus propias motivaciones y es transparente de un extremo al otro, porque elimina todo tipo de entidades, tales como imágenes, emociones, impulsos, sujeto trascendental, que enturbian la espontaneidad absoluta de la conciencia. Por eso, Sartre considera que la conciencia es "un absoluto" de la existencia, ya que no puede ser originada a partir de algo exterior. Y, en ese sentido, cabe afirmar que la conciencia es total transparencia, pues no hay nada, ni fuera ni dentro de ella, que la condicione. Sartre pone como ejemplo una caja de cristal transparente que deja pasar todos los vientos, siendo ella siempre traslúcida y abierta (2009, p. 16). O en palabras del mismo Sartre (1984): La conciencia es la realización de una posibilidad: surge en el seno del ser, crea y sostiene su propia esencia. La conciencia existe por sí y no tiene causa. La existencia pasiva, la del sujeto que no actuase, es impensable. Renunciando a la primacía del conocimiento, hemos descubierto el ser del cognoscente y encontrado lo absoluto. Un absoluto, no Substancial (1984, p. 13). Por tanto, se entiende "por absoluto no sustancial" que no hay nada en el interior de la conciencia, ni nada que la condicione.
} 
necesaria de ella, el yo terminaría convirtiéndose en un rango de absoluto para la conciencia. En definitiva, el yo tiene que ser un "objeto" de la conciencia y no una estructura necesaria de ella, ya que si lo es se convierte en un absoluto y, por ende, se convierte en una monada (Cfr. Sartre, 1966, pp. 25-26). Aclarando que cuando Sartre refiere al yo como "objeto" de la conciencia no lo está haciendo en categorías clásicas duales, de sujeto-objeto, más bien, al referirse al yo como "objeto", lo que está diciendo es que para que se cumpla el principio de la fenomenología — toda conciencia es conciencia de algo- es necesario que la conciencia apunte a un ser que no es ella misma (para este caso será el yo lo que ella está apuntando), y cuando se agote en el yo, es decir, cuando sea conciencia del yo, será un momento absoluto de la conciencia, puesto que el yo no hará parte de la conciencia, ni será una estructura de ella, solo habrá conciencia del yo.

A continuación, se explicará cómo la conciencia se "agota" y descubre a este "yo"; o, en otras palabras, se explicará cómo la conciencia por su intencionalidad toma conciencia del yo. Para ello se deberá analizar los dos momentos de la conciencia que Sartre desarrolla en la Trascendencia del Ego: la conciencia irreflexiva y la conciencia reflexiva.

\subsection{El cogito como conciencia reflexiva}

Como se dijo en la introducción de este trabajo, Sartre se distancia de la tradición filosófica; con ello no se quiere decir que prescinda de los conceptos o categorías que son propios de la misma tradición; a diferencia de autores que también han hecho una confrontación a la tradición filosófica, pero han preferido prescindir de conceptos o términos propios de la ontología clásica ${ }^{14}$. Una de estas categorías a las que Sartre decide dar continuidad es la de "cogito". Autores como Moran y Montero encuentran, por ello, en el pensamiento sartriano cierto cartesianismo ${ }^{15}$. Sin embargo, se entenderá este cartesianismo no como una continuidad en la concepción del cogito en Descartes, sino como una reivindicación del mismo. Pues según Sartre, Descartes cae en un error sustancialista. Este error consiste en haber interrogado al cogito sobre su aspecto funcional, a través del "pienso", e inmediatamente, sin ningún tipo de hilo conductor, Descartes salta de la interrogación sobre el aspecto funcional del cogito a un plano existencial: "luego-existo" (Cfr. Sartre, 1943, p. 109). Esto quiere decir que, en el pensamiento cartesiano, según Sartre, no hay una verdadera comunicación entre el cogito y el

\footnotetext{
${ }^{14}$ Como es el caso de Heidegger, que a pesar de haber sido discípulo de Husserl y contribuido al estudio de la fenomenología, evita, en lo posible, usar las palabras "conciencia" y "Yo".

Moran (2011) afirma en su libro Introducción a la fenomenología que el punto de partida de Jean Paul Sartre es el cartesianismo, "un cartesianismo mediado por la perspectiva idealista de sus maestros, especialmente León Brunschvicg” (p. 334). Por su lado Montero declara que el eslabón vinculante entre Descartes y Sartre es Husserl, debido a la influencia de la fenomenología husserliana en el pensamiento sartriano. Sin embargo, para Montero (1980) el cartesianismo de Sartre será más sutil que el de su maestro Husserl (Cfr. p. 328).
} 
plano existencial. Si no existe tal comunicación se está cayendo en un solipsismo. Por tal razón, la reivindicación que hará Sartre del cogito será la sustentación de una verdadera comunicación del cogito con el plano existencial. Para que se dé tal comunicación será necesario que el filósofo francés sustente al cogito como una "conciencia reflexionante". Lo que sigue a continuación es la explicación de por qué Sartre propone al cogito como una conciencia reflexionante.

Como se mostró en el anterior punto, a Sartre le interesa tratar la categoría del yo desde una cuestión de hecho; esto indica que para él el cogito será personal ${ }^{16}$; el mismo autor no los confirma cuando dice: "Ahora bien, es innegable que el cogito es personal" (Sartre, 1990, p. 43). Ya que si se trata de una cuestión de hecho sobre el cogito, se está hablando es desde mi experiencia personal donde lo descubro. Por ejemplo, quiero recordar aquella tarde que fui a cine con María; me es posible recordar esta escena, con muchos de sus detalles, pero también puedo acordarme que yo veía esa película ${ }^{17}$ (Sartre, 1966, p. 27). Con este ejemplo Sartre quiere señalar que el pensamiento, en una experiencia de rememoración, descubre al yo como personal, en la medida en que "puedo acordarme que yo veía esa película", que era precisamente yo el que la veía. Asimismo, al ser una experiencia de rememoración en la que se descubre al yo, se trata de una experiencia trascendental, porque el recuerdo, al que la conciencia se dirige, hace parte de una experiencia existencial vivida, no es una mera representación mental, se trata de un hecho concreto del mundo.

Por lo tanto, en cualquier rememoración que haga en modo personal aparecerá de inmediato el yo. Sartre (1990) dirá al respecto: "Tal es la garantía de hecho de la afirmación kantiana de derecho. Parece, pues, que no hay una sola de mis conciencias que no capte yo como estando provista de un yo" (pp. 43-44). Al presentar esta situación parecería que nos viéramos envueltos en un embrollo, pues como se dijo antes, el yo no debe ser una estructura necesaria de la conciencia. Sartre logra salir sin mayor dificultad de esta situación al recordar que todos los autores que han trabajado el cogito lo han presentado como una operación reflexiva, es decir, una operación de segundo grado ${ }^{18}$. Se trata de una conciencia que se dirige o toma como objeto a otra conciencia; en otras palabras, es una conciencia reflexionante (cuando se reflexiona sobre cualquier eventualidad del pasado) que descubre al yo como conciencia reflejada (yo leyendo, yo escribiendo, etc.). Según Sartre (1990) se está "en presencia de

\footnotetext{
${ }^{16}$ Cuando se dice que el cogito es personal, no se está haciendo referencia a una abstracción del termino de lo que significaría el ser persona, o como el personalismo de Martin Buber. Más bien se entiende por "personal" una experiencia concreta de cada individuo en relación con su pensamiento. Por ejemplo, yo pensando sobre alguna situación concreta de mi vida: yo leyendo, yo estudiando, etc.

${ }^{17}$ Sartre (1990) dice que a esta situación Husserl la llama la conciencia interna del tiempo, la posibilidad de reflexionar en el recuerdo (p. 43).

${ }^{18}$ Para Sartre la conciencia reflexiva será una operación de segundo grado o conciencia de segundo grado, pues depende de otra conciencia, es decir, de la irreflexiva o conciencia de primer grado.
} 
una síntesis de dos conciencias, una de las cuales es conciencia de la otra. Se salvaguarda así el principio esencial de la fenomenología: 'toda conciencia es conciencia de algo'" (p. 44).

En este sentido, cabe aclarar qué entiende Sartre por esta conciencia de conciencia. En El ser y la nada rechaza la idea de que está conciencia de conciencia sea la primacía del conocimiento como, conocimiento de sí; esto quiere decir que para que yo pueda saber o conocer el computador que tengo al frente mío, es necesario que sea consciente de estar conociendo este ordenador; o en palabras de Alain, citado por Sartre (1984), "saber es saber que se sabe" (p. 22). La razón por la cual Sartre rechaza esta definición de la conciencia de conciencia se debe a que, en primer lugar, se entendería la conciencia como una dualidad $^{19}$ (cognoscente-conocido). Esto a su vez introduce un problema mayor, pues el cognoscente deberá ser conocido por otro cognoscente, y este a su vez por otro; esta situación nos presenta un dilema, pues en algún punto de la serie cognoscente-conocido-cognoscente... se habrá que detener y reconocer que hay un punto de la reflexión no consiente de sí o afirmar la necesidad de hacer una regresión al infinito, lo cual para Sartre es absurdo (Cfr. 1984, p. 22).

Otro problema que se presenta en definir a la conciencia de conciencia como conocimiento de sí es que la conciencia reflexiva debería estar permanentemente presente para que fuera constitutiva de toda conciencia reflejada, de lo contrario el conocimiento no sería posible. Al poner a la conciencia reflexiva como condición del conocimiento, se está regresando a una postula idealista. Por tales razones Sartre rechaza esta interpretación de la conciencia, pues como se mencionó al principio de este escrito, el planteamiento sartriano sobre la conciencia es evitar caer en cualquier tipo de idealismo o dualidad. Para Sartre (1984) la conciencia de conciencia es una conciencia espontánea propia de mi percepción y constitutiva de mi conciencia perceptiva (p. 22). Es decir, en el momento que me pongo a contar los cigarrillos que tengo en mi bolsillo y descubro que son doce, Sartre dirá que este resultado, que es una propiedad objetiva del mundo, se me revela por un acto espontáneo de conteo y no porque en el momento de hacer la operación me esté viendo a mí mismo contándolos por un acto de reflexión. Cuando reflexiono sobre la operación que acabo de hacer, me doy cuenta de que en ese instante, en el que contaba cada cigarrillo, había una conciencia no tética ${ }^{20}$ de mi actividad, una conciencia irreflexiva que finalmente fue la que posibilitó que pudiera hacer la suma (Cfr. Sartre, 1943, pp. 18-19). En definitiva, la conciencia de conciencia es una conciencia irreflexiva que permite la reflexión. Sartre (1984) lo confirma

\footnotetext{
${ }^{19}$ Recuérdese que la concepción que está buscando Sartre para la conciencia evita caer en cualquier tipo de dualidad.

${ }^{20}$ Para Sartre la conciencia no tética o no posicional es cuando el individuo ignora que existe, debido a que se encuentra absorto en cualquier tipo de actividad, ya sea leyendo, manejando bicicleta, comiendo, etc. En otras palabras, no se está reflexionando sobre la actividad que se hace, es decir, no se está viendo así mismo ejercerla, simplemente se hace (Cfr. Sartre, 2016, p. 359).
} 
cuando dice "hay un cogito prerreflexivo que es la condición del cogito cartesiano" (p. 22).

Así introduce el autor la distinción entre un cogito reflexionante y un cogito irreflexivo, correspondientes a una conciencia reflexionante e irreflexiva. El cogito, dice Sartre, en función reflexionante e irreflexiva, la conciencia, en estado reflexivo o irreflexivo. El cogito es, en sentido estricto, reflexionante y no reflexivo, porque no es un cogito "posicional", es decir, no convierte al yo en un "objeto" de su reflexión. Él es re-flexivo no por un acto de toma de posición de sí mismo, sino porque para él reflexionar es inmediatamente entrar en contacto con lo que él no es, en una experiencia que va más allá de sí mismo. La conciencia reflexionante no puede ser objeto para sí misma porque el yo que piensa "en" ella se encuentra ocupado con las cosas, fuera de sí. Ella logra ser conciencia posicional solo cuando toma como objeto de su reflexión la conciencia irreflexiva. De este modo Sartre (1990) concluye: "La conciencia que dice "yo pienso" no es, precisamente, la que piensa. $\mathrm{O}$, mejor: no es su pensamiento lo que ella pone mediante este acto tético" (pp. 44-45).

Ahora bien, ¿̇cuál es la ubicación del yo entre la conciencia reflexiónate y la irreflexiva? El problema implicado aquí lo plantea Sartre de esta manera: ¿cómo podemos hablar de un yo irreflexivo, de una conciencia irreflexiva, si siempre que recurro a mis experiencias estas se encuentran acompañadas del yo? como, por ejemplo, cuando acudo a una experiencia de leer aparece la conciencia de "yo leo" como experiencia reflexiva. ¿Cómo hago para escapar del yo si cuando acudo a la experiencia me veo "yo leyendo", "yo manejando bicicleta", "yo escribiendo", etc.? Sartre (1990) puede salir de este apuro al sostener que "siendo esta conciencia no-tética de sí misma, deja un recuerdo no-tético que sí se puede consultar" (p. 46). Se debe aclarar que "no tético" significa no "puesto", no "fijado" por el pensamiento, a modo de una tesis o proposición. Volvamos con el ejemplo del cine para dar mayor claridad a lo expresado. Hace unos días fui al cine, estaba viendo la película y me encontraba absorto entre los personajes de la película, sus diálogos, vestuarios, etc. Al revivir este recuerdo me puedo dar cuenta de la existencia de la conciencia irreflexiva. No cabe duda de que en ese momento había conciencia del héroe de la película, del asiento donde me ubicaba, de mi compañera. Pero justo como una conciencia no tética, porque al recuerdo al que acudo es un recuerdo no tético, un recuerdo en el que ese día estaba "viendo la película", y no un recuerdo de que estaba reflexionado o viéndome a mí mismo viendo la película (Sartre, 1966, p. 30).

El interés de Sartre al plantear toda esta situación, es el de concluir que en el plano irreflexivo no es posible la existencia de un yo tético, posicional, ya que la conciencia se encuentra sumergida en el mundo de los objetos, son ellos los que constituyen la unidad de mi conciencia. No hay, advierte Sartre, espacio para mí, en el sentido de un yo posicional que acompañe todas mis experiencias, 
pues como conciencia irrefleja no estoy pensando o analizándome como "yo tomando el bus", "yo leyendo" "yo comiendo"; lo que hay es "conciencia de leer", "conciencia de comer", "conciencia de tomar el bus" (Sartre, 1966, p. 32).

Me gustaría resaltar, finalmente, algunas de las conclusiones a las cuales llega Sartre respecto a la relación del yo frente a la conciencia. En primer lugar, la existencia de un yo reflexivo se parece más a la de las verdades eternas, ya que este no se encuentra inmerso en una experiencia concreta, haciendo parte de la estructura perecedera de esta, debido a que funciona como su sustrato o fundamento intemporal, garantía de su verdad. El yo no es, como se mostró, fuente de la conciencia, sino que ella es "fuente" de sí misma, en la medida en que no necesita de una realidad o estructura interna para poder fundamentarse. Vale aclarar que ser "fuente de sí misma" no significa que, de acuerdo con lo expuesto hasta aquí, la conciencia no necesite del mundo para existir, sino que, por el contrario, alude a su ser esencialmente exterior: la conciencia existe en el mundo. El yo para Sartre, de este modo, posee una existencia irreflexiva al presentarse como algo que no es fuente de la conciencia. Este hecho pone de manifiesto la realidad opaca del yo, esquiva a los actos de reflexión que buscan objetivarlo.

En segundo lugar, si el yo hiciera parte de la conciencia, estaríamos hablando de dos yoes: el de la conciencia reflexiva y el de la conciencia irreflexiva. Este problema de la existencia de dos yoes es inconcebible para Sartre, ya que sería admitir una comunicación entre el yo reflexivo y el yo irreflexivo, en la que aquel, el yo reflexivo, termina legitimando al yo irreflexivo. No hay comunicación, ni mucho menos una identificación, entre ellos. Afirmar lo contario es ignorar que siempre hay dos formas de existencia posible para el yo y para la conciencia: la vida irreflexiva y la vida reflexiva. La confusión radica en negar esta distinción y suponer o una conciencia reflexiva en la conciencia que se da como irreflexiva o proponer una estructura reflexiva en la conciencia que permanece irreflexiva (Sartre, 1966, pp. 36-37).

En cuanto a la relación entre el cogito y la conciencia, se concluye con la afirmación que ofrece Sartre (2016) en su conferencia Conciencia de sí y conocimiento de sí: "En efecto, el cogito no es más que la manifestación de la conciencia" (p. 348). Lo es, debido a que el cogito es una conciencia reflexionante, pues toma como objeto a otra conciencia, es decir, cuando se piensa, el contenido de este pensamiento es sobre algo, pero este algo que toma por objeto el cogito, lo pensado, es la conciencia irreflexiva o cogito prerreflexivo, que últimas es su soporte; el mismo Sartre (2016) lo afirma: "Hay un "cogito" prerreflexivo que es la condición del "cogito" cartesiano" (p. 348). Este cogito prerreflexivo corresponde a la conciencia irreflexiva, pues es la conciencia o vivencia que aún no se ha 
tomado por reflexión, (hay conciencia de manejar bicicleta ${ }^{21}$, hay conciencia de leer, hay conciencia de pensar, etc.). En definitiva, el cogito cartesiano al tener como soporte un cogito prereflexivo supera el reproche que se le ha hecho de haber caído en un solipsismo ${ }^{22}$, pues en últimas dicho soporte es el mundo mismo.

Cabe señalar la importancia de diferenciar el cogito prereflexivo del cogito reflexivo, pues de lo contrario, se caería en un error metodológico frente a la lectura que se haga sobre la conciencia y su relación con el yo. A continuación, se explicará en que consiste tal error.

\section{2: Teoría de la presencia material del Mí mismo}

En la tercera parte del primer capítulo de la Trascendencia del Ego Sartre argumenta en contra de la teoría del "amor propio", que para él es puramente psicológica ${ }^{23}$. Dicha teoría propone al "mí mismo" como presente en todos nuestros estados de conciencia. Esto quiere decir que quienes proponen esta teoría consideran que el amor de sí, y por tanto el mí mismo, se encontraría disimulado u oculto, en todos mis actos y sentimientos, por un principio egoísta que me mueva a satisfacer un deseo oculto. Esto hace que El "mí mismo" se convierta en una estructura esencial para cada uno de mis actos, ya que en últimas lo que impulsaría a todos mis actos sería un deseo egoísta o amor propio por mi persona. Si esta teoría es veras, el "mí mismo" sería constitutivo de toda conciencia (Sartre, 1966, p. 38).

Para dar más detalles sobre esta teoría del "amor propio" Sartre menciona a La Rochefoucauld ${ }^{24}$. Según Sartre, fue él uno de los primeros que hizo uso del inconsciente, sin mencionarlo propiamente. La Rochefoucauld afirma, según

\footnotetext{
${ }^{21}$ El contenido del Cogito como impersonal, Según Bard, es una de las tesis centrales del pensamiento Sartriano (Cfr. 2002, p. 9). Por contenido impersonal debe entenderse que para Sartre el contenido del Cogito no es "yo tengo conciencia de esta silla", sino "hay conciencia de esta silla" (Cfr. Sartre, 1990, p. 53), es decir, no es el resultado de un acto reflexivo, por el contrario, es la vivencia misma quien le da contenido al Cogito.

${ }^{22}$ Los reproches que se acostumbran hacerle al "cogito", en tanto él es tomado como base apodíctica de una filosofía, son los siguientes: [...] Él no permite salir del solipsismo, a menos que se recurra a la idea de Dios, es decir, a menos que se haga el "salto" ontológico [...] (Sartre, 2016, p. 347).

${ }^{23}$ En este capítulo Sartre critica el inconsciente, entendido como esa fuerza oculta, la cual hace que todos mis actos se deban a un principio egoísta o de "amor propio". Es decir que todo lo que hago se deba a satisfacer un deseo y no lo haga por el simple albedrío.

${ }^{24}$ Su nombre completo es Francisco VI, duque de La Rochefoucauld, nació en París el 15 de septiembre de 1613 y murió el 17 de marzo de 1680. Fue conocido, sobre todo, por su escrito Reflexiones o Sentencias o Máximas morales, al que seguramente Sartre está haciendo referencia, pues en cada reflexión que hace La Rochefoucaul en su libro se sustenta que todo acto del ser humano está impulsado por el amor propio que se tiene a sí mismo y no por ningún tipo de altruismo o virtud que pueda existir en el ser humano. Por ejemplo, en la reflexión número 53 Rochefoucauld (1824) afirma: "El apego o la indiferencia de los filósofos a la vida no es más que una complacencia de su amor propio; de que debemos disputar tanto como del gusto del paladar, o de la elección de los colores" (p.16).
} 
Sartre, que el amor propio se disimula bajo las formas más diversas, pues el "mí mismo", si no está detrás de la conciencia, está oculto, como un "imán de atracción" de todas nuestras representaciones y de todos nuestros deseos. En otras palabras, es el "deseo" el que se da como fin o motor de la conciencia (Sartre, 1966, p. 38).

La objeción que presenta Sartre (1990) sobre esta teoría, consiste en que para él los psicólogos promotores de dicha presunción confunden "la estructura esencial de los actos reflexivos con la de los actos irreflejos" (p. 55). Se está ignorando, con esto, que siempre hay dos formas de existencia posible para una conciencia: la conciencia irreflexiva y la conciencia reflexiva. La confusión radica en negar esta distinción y suponer o una conciencia reflexiva en la conciencia que se da como irreflexiva o proponer una estructura reflexiva, que con descuido se creé que permanece inconsciente. Un ejemplo de lo dicho puede ser: María necesita un consejo y decido aconsejarla, en ese momento solo existe para mi conciencia "debo aconsejar a María" y ella es "objeto" que atrae toda mi atención y no mi "amor propio". Al decir que ella es "objeto", se está diciendo que ella hace parte de un mundo real, un mundo cuyo sentido no es creado por mi conciencia (como lo propondría cualquier teoría idealista), sino que hace parte de su propia realidad, está, de este modo, por fuera de mi pensamiento, y por ello reclama toda mi atención. Al respecto Sartre (1990) afirma: "Hay un mundo objetivo de cosas y acciones, hechas o por hacer, y las acciones vienen a aplicarse como cualidades sobre las cosas que reclaman" (p. 56). Esto quiere decir que la conciencia irrefleja no constituye o determina el sentido objetivo del mundo, sino que ella percibe el mundo directamente como algo real u objetivo. Al ser el mundo real y objetivo, este reclama una participación de mi conciencia; para este caso, si María necesita ayuda, voy y la ayudo de manera espontánea, no porque me he inventado que ella necesita de mi ayuda, sino porque realmente la necesita.

Cabe aclarar que este momento en el que ayudo a María, aparte de ser un momento de la conciencia irrefleja también es un momento espontáneo; lo es porque no está mediado por la reflexión. Al no ser un momento mediado por la reflexión, no se puede admitir la suposición de la existencia de una intención oculta o egoísta que me motive a aconsejar a María. Este estado de conciencia se descubre en el hecho mismo de aconsejar a María. Dicho en otros términos, la acción misma de aconsejar a María es la conciencia de aconsejarla. Sin embargo, los psicólogos que apoyan la teoría del "amor propio" no lo ven así, pues Sartre dirá (1990) "han imaginado tras él otro estado que queda en la penumbra" (p. 56). A diferencia de los psicólogos que apoyan la teoría del "amor propio", Sartre considera que este estado de querer aconsejar a María no oculta algo de tras de sí que pueda ser conocido como tal por un estado de reflexión. Por el contrario, debo saber qué le acontece a María para desear aconsejarla y no suponer que existe algo oculto que se encuentra detrás de mi conciencia. Además, Sartre (1990) 
añadirá: "La conciencia irrefleja debe ser considerada autónoma. Es una totalidad que no tiene necesidad alguna de ser completada, y debemos reconocer, sin más, que la cualidad del deseo irreflejo es trascenderse al captar sobre el objeto la cualidad deseable" (p. 57).

De esta manera Sartre concluye que en el plano irreflejo se sitúa la vida impersonal y en el plano reflexivo se ubicaría la vida egoísta. Por ejemplo: aconsejo a María porque ella es alguien que "debe ser aconsejada". Hasta este punto se está en el plano irreflejo, pues en un acto espontáneo voy y aconsejo a María. Se trata de un acto espontáneo porque "hay conciencia de estar aconsejándola" y en ningún momento estoy reflexionando acerca de lo que hago o no me estoy viendo a mí mismo aconsejándola. Si de repente en ese instante me veo aconsejando a María y me veo hablándole a ella, puede que me interrogue si lo que hago este bien; si concluyo que lo está, podría continuar o quizás me quede callado, porque me conviene hacerlo, sin importar la ayuda que mis palabras podrían ofrecerle. En ese instante aparece mi actitud egoísta, que se da desde la reflexión y no como un acto espontáneo. Sartre (1990) afirma al respecto: "El Mí mismo no aparecerá más que con el acto reflexivo y como correlato noemático de una intención reflexiva" (p. 59).

Se concluye, para este punto, que el "mí mismo", al igual que el yo, es objeto para la conciencia, es decir, no yace en su interior. El error radica, para quienes han creído lo contrario, según Sartre, en no diferenciar los dos momentos que tiene toda conciencia: reflexivo he irreflexivo. Pues, como se mencionó y según el análisis del filósofo francés, el deseo o impulso egoísta aparecen solo mediante un acto de reflexión y no de manera espontánea.

\section{Conclusiones}

Luego de haber expuesto y analizado el problema del yo y la conciencia en la filosofía sartriana, se llegaron a las siguientes conclusiones: Sartre está de acuerdo con Husserl en que la trascendencia es el ser de la conciencia, o en otras palabras, que toda conciencia es conciencia de algo ${ }^{25}$. Sin embargo, Sartre no está conforme con la argumentación que ofrece Husserl respeto a la trascendencia de la conciencia ${ }^{26}$, por ello, el autor francés, diseña todo un aparato argumentativo a favor de su noción de trascendencia. Sartre comparte con Husserl la idea de

\footnotetext{
25 "La conciencia es conciencia de algo: esto significa que la trascendencia es estructura constitutiva de la conciencia; es decir, que la conciencia nace apuntando hacia un ser que no es ella misma" (Sartre, 1984, p. 29).

${ }^{26}$ Pero, se dirá, Husserl define precisamente la conciencia como una trascendencia. En efecto: tal es su tesis, y su descubrimiento esencial. Pero, desde el momento en que hace de la noema algo irreal, que es correlato de la noesis y cuyo esse es un percipi, se muestra totalmente infiel a su principio (Sartre, 1984 , p. 30).
} 
que la conciencia no es algo "en sí mismo", en el sentido de una conciencia que se define por su relación consigo misma, por ser conciencia de sí misma. La conciencia siempre será para Sartre y así mismo para Husserl, "intencional"27, es decir siempre será conciencia "de" "algo" que se encuentra en el mundo: hay conciencia de estudiar, hay conciencia de leer, hay conciencia amar, etc. Pero para Sartre, a diferencia del filósofo alemán, esta idea de la intencionalidad equivale a concebir a la conciencia como "exterioridad pura". Esta fue la tesis que se sustentó a lo largo del presente trabajo. La conciencia es "exterioridad pura": esto significa que para Sartre la conciencia tiene un carácter de "vacuidad" 28 , y que la única forma para que esta pueda existir es que sea conciencia de algo que se encuentre en el mundo.

Así mismo, para Sartre no hay nada interior en la conciencia, incluso las categorías "psíquicas" como el yo y el moi están por fuera de la conciencia. Lo anterior se vio reflejado en el planteamiento de la imposibilidad de la existencia del yo como compañero permanente de cada estado de conciencia, pues se mostró que hay momentos de la conciencia donde no aparece el yo, y que la afirmación kantiana que dice "el yo pienso debe poder acompañar a todas nuestras representaciones", solo se concibe desde una cuestión de derecho. Otro argumento que contribuyó, dúrate esta exposición, a sustentar la vacuidad de la conciencia, fue la refutación hecha por Sartre, a la teoría del amor propio, la cual afirma que toda acción está determinada por un deseo de satisfacción que se encuentra oculto en la conciencia, en síntesis, la refutación que se presentó fue que todo impulso egoísta necesariamente aparece desde la reflexión.

Al no haber "nada" en el interior de la conciencia se concibe que la única forma en que la conciencia pueda existir es que sea conciencia de "algo", es decir que sea conciencia intencional del mundo. Para Sartre si el yo o el moi se encuentran en el interior de la conciencia significaría la muerte de la misma, ya que al ser el yo un habitante de la conciencia, esta dejaría de ser intencional, pues su fundamento no sería el mundo sino el yo. De ser así, no sería posible una comunicación entre la cosa pensante (el individuo) y la existencia (el mundo), se cometería, en palabras de Sartre, un error sustancialista, es decir se regresaría a una postura solipsista ${ }^{29}$. Por esta razón el yo no debe estar en el interior de la conciencia ni detrás de ella, su lugar, donde se encuentra, es el mundo. Finalmente,

\footnotetext{
27 Al respecto añade Butler (1987) La direccionalidad de la conciencia, su tendencia hacia las cosas exteriores a ella, viene a expresar la situación ontológica de los seres humanos como una "espontaneidad" y un "desdoblamiento". En cuanto seres intencionales, no sólo nuestro conocimiento es del mundo, sino también nuestra pasión esencial; nuestro deseo es quedar cautivados con el mundo, ser "del" mundo. La intencionalidad viene a representar el acceso humano al mundo, el fin de las teorías que aíslan de este último a la conciencia y la subjetividad y las obligan a residir detrás de la densa cortina de la representación (p. 156).

${ }^{28}$ Es decir, la falta de contenido interno en la conciencia.

${ }^{29}$ Al final de la Trascendencia del Ego el mismo Sartre (1990) reconoce que "Esta concepción del Ego nos parece la única refutación posible del solipsismo" (p. 108).
} 
la tesis sartriana de la exterioridad de la conciencia, viene a representar la separación de la filosofía de Sartre con la tradición filosófica, concretamente con un idealismo que ha propuesto al individuo como creador de la realidad y con un solipsismo que solo ha sido capaz de justificar la comunicación entre interioridad y exterioridad por medio de un ente trascedente. Sin duda, el aporte que hace Sartre a la fenomenología y concretamente al estudio de la conciencia, es de gran valor para la construcción de nuevas lógicas del pensamiento.

\section{Referencias}

Álvarez, L. G. (2009). Sartre: La conciencia como libertad infinita. Tópicos, (37), 9-29.

Bard, X. (2002). Pour une Lecture Critique de la Transcendance de L'ego. Paris: L'Hannatlan.

Butler, J. (1987). Sujetos del deseo. Buenos Aires: Amorrortu Editores.

Husserl, E. (2013). Ideas relativas a una fenomenología pura y una filosofía fenomenológica. (I. A. Zirión Quijano, Trad.). Ciudad de México: Fondo de Cultura Económica.

Kant, I. (2011). Crítica de la Razón Pura. (M. García Morente, Trad.). Madrid: Editorial Tecnos.

Koffi, A. (2013). Heidegger et Sartre: Quelles Conceptions de L'être? Revue Spécialisée en Études Heideggériennes, (1), 71-89.

La Rochefoucauld, F. (1824). Reflexiones o sentencias y máximas morales. (N. Alvaro y Zereza, Trad.). París: Casa de Masson e Hijo.

Montero, J. (2007). La Fenomenología de la Conciencia en E. Husserl. Universitas Philosophica, 24(48), 127-147.

Montero, F. (1980). La Fenomenología del "Yo" de Jean-Paul Sartre. Teorema: Revista Internacional de Filosofía, 10(4), 327-346.

Morán, E. (2011). Introducción a la fenomenología. Buenos Aires: Serrano Editores.

Sartre, J. P. (1943). L'ête et le néant. Paris: Éditions Gallimard.

Sartre, J. P. (1966). La Trascendance de L'ego. Paris: Librairie Philosophique. 
Sartre, J. P. (1984). El ser y la nada. (J. Valmar, Trad.). Madrid: Alianza editorial.

Sartre, J. P. (1990). La Trascendencia del Ego. (M. García-Baró, Trad.). Madrid: Editorial Síntesis.

Sartre, J. P. (2016). Conciencia de sí y conocimiento de sí. Acta fenomenológica latinoamericana, (V), 343-371.

Vélez, D. C. (1970). Filosofía sin supuesto. Buenos Aires: Editorial Suramericana. 